\title{
Research on Inventory Control Policies for Nonstationary Demand based on TOC
}

\author{
Leng Kaijun* \\ Research center of Hubei Logistics development, Hubei University of Economics, \\ No.8,YangQiaohu road,Jiangxia Canglong development zones, Wuhan, 430205, China \\ Wang Yuxia \\ Department of Information and Engineering, Wuhan University of Technology Huaxia College, \\ No.589, Guanshan road, East lake high-tech industrial development zone, Wuhan, 430223, China \\ E-mail: Wangyuxia0318@163.com \\ www.hbue.edu.cn
}

\begin{abstract}
An effective inventory replenishment method employed in the supply chain is one of the key factors to achieving low inventory while maintaining high customer delivery performance. The state of demand process is often not directly observed by the decision maker. Thus, in many literatures, the inventory control problem is a compositestate, partially observed Markov decision process (POMDP), which is an appropriate model for a number of dynamic demand problems. In practice, managers often use certainty equivalent control (CEC) policies to solve such a problem. However, in reality, Theory of Constraints (TOC) has brought a practical control policy that almost always provides much better solutions for this problem than the CEC policies commonly used in practice. In this paper, we proposed three different inventory control policies based on TOC buffer management framework, and use simulation approach to compare them with traditional adaptive (s,S,T) policy. The computational results indicate how specific problem characteristics influence the performance of whole system and demonstrate the efficiency of the proposed control policy.
\end{abstract}

Keywords: Inventory control policy; Nonstationary demand; Theory of constraints; Buffer management

\section{Introduction}

A supply chain is a set of nodes which consist of production plants, central warehouse, regional warehouses and points of sales. The chain links supplies and customers, beginning with the production of products by a supplier, and ending with the consumption of a product by the customer ${ }^{1}$. The need for regional warehouse stems from the need to supply the market very quickly. In the business market of flaming competition in recent years, companies are plagued by the fluctuations in demand and inevitably present inventory management challenges of the right inventory in the right place (node) at the right time. An effective inventory replenishment method employed in the supply chain is one of the key factors to achieving low inventory while maintaining high customer delivery performance.

Most inventory control problems exist in situations in which demand is both nonstationary and partially observed. By nonstationary, we mean that the probability distribution of the demand changes over time. Furthermore, the true underlying distribution of the demand in a period is never directly observed; it is only partially observed through the actual demand values. Under these conditions, inventory managers estimate the unknown demand distribution and typically use some

* Corresponding author. Tel.: +86-027-81973740; Fax: +86-027-81973739. E-mail address: kaijunlen@yahoo.com.cn (Leng Kaijun) 
type of certainty equivalent control (CEC) policywhich assumes the estimate is correct-to determine an inventory stocking level. For example, a manager may forecast the demand mean and variance over future time periods and use these estimates in a traditional inventory model to set stocking levels. The results presented in this paper demonstrate that there exist other practical suboptimal control policies to solve realistic instances of this problem without assuming such an estimate. Furthermore, these control policies achieve much better performance than the CEC policies commonly used in practice in almost all the cases that were considered. These suboptimal policies include Open-Loop Feedback Control and Limited Look-Ahead Control. Both of these policies account for more of the inherent uncertainty in the demand process and, therefore, almost always achieve lower expected cost than the CEC policies.

However, an effective replenishment method should resolve the following three basic issues: (1) how often the inventory status should be determined. (2) when a replenishment order should be placed. and (3) how large the replenishment order should be. Replenishment methods proposed in the traditional inventory theory can be classified as either continuous review systems ((s, S) or $(\mathrm{s}, \mathrm{Q})$ policy) or periodic review systems $((\mathrm{R}, \mathrm{S})$ or $(\mathrm{R}$, $\mathrm{s}$, S) policy) $)^{2}$. However, researches report that order based on these replenishment methods swing due to downstream supply chain partners' fluctuation of demand. The swing is amplified as the order moves up to the supply chain. This phenomenon of demand amplification is named as bullwhip effect. Bullwhip effect causes excessive inventory, loss of revenue, and inaccurate production plans throughout supply chain systems $^{3}$. The improvement of bullwhip effect in a supply chain is a key challenge for a manager. The Theory of Constraints-supply chain replenishment system (TOC-SCRS) is one of the solutions for the improvement of the bullwhip effect in a multi-echelon supply chain ${ }^{4}$.

However, when the TOC-SCRS is applied in a plant or a central warehouse, the determination of RRT will encounter a conflict with the replenishment quantity. That is because the replenishment frequency depends on the set up frequency in the plant and the replenishment lead time depends on the production lead time. As we know, however, the set up frequency and the replenishment lead time in the plant depend on the production quantity (i.e., replenishment quantity). It means that the replenishment frequency and the replenishment lead time must depend on the known replenishment quantity, especially under the constraint of limited plant capacity. However, in TOC-SCRS, the replenishment quantity depends on the known parameters of the replenishment frequency and the replenishment lead time.
Therefore, this is a big conflict and an issue to apply the TOCSCRS in a plant or a central warehouse. An enhanced replenishment model for TOC-SCRS under capacity constraint is then required to be provided to solve this conflict ${ }^{5}$. Although TOC-SCRS concept has been implemented by a growing number of companies, its model is not described in the literatures. The model of TOC-SCRS based on previous research ${ }^{6}$ is then modeled in this paper first. Then three different enhanced inventory control policies for TOC-SCRS under capacity constraint is then developed. A simulation comparison and its sensitivity analysis are utilized to evaluate the application of the proposed method.

\section{Literature review}

The inventory control literature can be roughly categorized into four classes. First, there are problems with fully observed and stationary demand that form the body of most of the classical inventory theory. Lee etc. summarize this classical theory quite well ${ }^{7}$. Second, there are problems with fully observed and nonstationary demand. These problems, although more complex, have been studied fairly well also, e.g., rare research is available for the third and fourth classes of problems, those with partially observed, stationary demand, and those with partially observed, nonstationary demand. This paper mainly focuses on the case of nonstationary demand particularly on the seasonal demand fluctuation, but first, we review literatures for these related problems.

One of the earliest cases of a stochastic nonstationary demand problem is found in Literature ${ }^{8}$ Shortly prior to that, Literature ${ }^{9}$ has analyzed a dynamic system (nonstationary and stochastic) in which the distribution of demand can vary each period, and it is an extension of the Arrow-Harris-Marshak dynamic inventory model, and the final section discusses cases of "partial information," in which an unknown parameter of the demand distribution has an a priori distribution and the distribution is stationary.

More recently, some literatures have discussed a general modeling framework in a fluctuating demand environment modeled by a Markov chain, in which demonstrated how inventories should be managed in the face of possible obsolescence using a similar demand model ${ }^{10}$. Although their demand model is similar to the one presented in this paper, the critical difference is that their model assumes that the current demand state is directly observed. Some has proposed an adaptive based stock inventory policy for a nonstationary problem in which the demand model is an integrated moving average.

Problems with partial information are significantly 
more difficult to solve. Stationary partial information problems may be solved by Bayesian methods or other adaptive procedures. Some scholar significantly extended the early results of Bayesian methods and some others uses a myopic parameter adaptive technique and a simple inventory policy based on a critical fractile, in which the demand model is similar to the one used in this paper, but in this case the critical difference is that the core process is stationary. Afterwards, some scholar extends this analysis of myopic policies by considering policies that terminate the myopic behavior at some point $\mathrm{T}$, which may be fixed or random. Then some scholars have provided additional insights into the stationary partial information problem.

Literature $^{11}$ demonstrate a Min-Max technique for analysis of various distribution-free finite horizon models for which the distribution is specified by a limited number of parameters such as the mean and variance or a set of percentiles of demand. Based on that, some scholar start consider Bayesian techniques for a lost sales problem in which sales, not true demand, are observed. Because of lost sales, the retailer can learn more about the true demand process by holding inventory at a higher level initially to quickly establish a better estimate of the true demand, and some other literatures have examined lost sales and contend that the negative binomial family provides a very good fit for actual retailing sales data. Problems with nonstationary demand and partial information present an even more complex situation. Little direct work has been done in this area, but, there is great potential impact for research on this class of problems. In summarize, there are many literatures presented a growth model to estimate the parameters of a demand process over its entire life cycle. In their base case, production decisions are made at the beginning of the problem for the entire life cycle, and some of them presented a technique to initially estimate the parameters of their forecasting model ${ }^{12}$. However, these issues still remain the same in reality.

In 1979, development of the Theory of Constraints (TOC) management philosophy began with the introduction of Optimized Production Timetables scheduling software ${ }^{13}$. TOC has evolved from this simple production scheduling software program into a suite of integrated management tools encompassing three interrelated areas: logistics/production, performance measurement, and problem solving/thinking tools ${ }^{14}$. Due to its simple yet robust methodology, application of TOC techniques have been discussed in the academic literature and popular press across a variety of operations management sub disciplines.

Studies reporting anecdotal evidence from early adopters suggested that TOC techniques could result in increased output while decreasing both inventory and cycle time $^{15}$. Rigorous academic testing has validated those early findings revealing that manufacturing systems employing TOC techniques exceed the performance of those using Manufacturing Resource Planning (MRP), Lean Manufacturing, Agile Manufacturing, and Just-in- Time (JIT). The results of these studies indicate ${ }^{16}$ that TOC systems produce greater levels of output while reducing inventory, manufacturing lead time, and the standard deviation of cycle time.

The TOC replenishment solution utilizes this fact, a solution that we have already. Hold the inventory at the most accurate point. This is not very accurate, but it is the most accurate point of the system. At the source-at the plant in most cases is the most accurate point of the system. That is where we have the biggest aggregation. TOC states ${ }^{6}$ that aggregating the inventory and holding it at the plant warehouse not only decouples production disruption from bullwhip effect, but also increases the reliability of the replenishment of the goods to the regional warehouse, by making the replenishment time equal to the transportation time only. Accordingly, hoarding inventory in the distribution chain is rendered unnecessary and other links need not attempt to overprotect themselves with excessive inventory. The goal of having the right inventory at the right place at the right time can thus be achieved.

The solution is effective. However, determining the right amount of buffer and performing inventory buffer control al the plant warehouse and regional warehouses remains an issue. Various mathematical methods have been applied to determine optimum buffer size. when demand rate varies with the time, the trigger rules should adjust to accompany the demand change. Silver etc uses rolling horizon of length $R+L$ (review period and lead time) approach that allows the demand rate vary within the current horizon by using safety stock considerations to decide when to place an order, followed by the deterministic Silver Meal heuristic to select the size of the then current replenishment ${ }^{17}$. In addition to Silver, many other scholars also addressed this problem as well. Some develops a stochastic version of the Wagner-Whitin model. However, these models have drawbacks from practitioner's standpoint. some suggest a heuristic method, known as the revised power approximation. A somewhat similar approach was presented in literature ${ }^{18}$ which have developed an accurate approximation for both $s$ and $Q$ when a fill rate is used. Their approximation can be built on a spreadsheet very easily. Some have developed a myopic heuristic, involved precomputing the $(s, S)$ values for various values of mean demand. However, this heuristic should be avoided if demand is expected to decline rapidly. Many others have developed a fast algorithm for finding the optimal $s$ and $S$ for given $R$ and for discrete demand distributions. However, this algorithm, 
using a programming language, is best developed on a computer rather than a spreadsheet. Based on this, many scholars have suggested some heuristic methods for determining the reorder point in the continuous review context for a product with unequal, discrete stock withdrawals under deterministic conditions. Research efforts in this regard have not been adequate to date. Literature $^{19}$ extended the study and use simuiation heuristic procedures to design mechanisms for triggering set-ups of replenishment. Additional related work on this problem includes literature ${ }^{20-22}$.

TOC buffer management is feasible and powerful, but is not sufficiently rigorous. A rigorous procedure must be developed to implement the method effectively in the real world, especially if the method is to be computerized. No such method is yet available in the literature. Therefore, this paper proposes a generic buffer management procedure, based on TOC buffer management that will define rigorously a monitoring approach for sizing the buffer, monitoring the buffer and adjusting it accordingly. A generic procedure is one that should be further tuned for application in a specific area. Furthermore, an simulation based comparison illustrates the feasibility of the proposed procedure.

\section{Basic Model and Nomenclature}

Prior to introducing the proposed policies and describe their detailed procedure, and for a better understanding of their exposition, in what follows we first present the nomenclature that is used throughout this paper and the basic model based on TOC.

Nomenclature:

$L_{Q} \quad$ Regular replenish lead time

$T \quad$ Order review period, which is the regular time interval between the reorder points. The buffer procedure regularly replaces the order at each order reorder point.

$L_{E} \quad$ Emergency replenishment time, which is the shortest time required to replenish the emergency order.

$R(t) \quad$ Order arrived quantity at $t$ period

$O_{Q}(t) \quad$ Regular order quantity at $t$ period

$O_{E}(t) \quad$ Emergency order quantity at $t$ period

$I T(t) \quad$ On-road stock quantity at $t$ period

$I L_{1}(t) \quad$ On-hand stock quantity at the beginning of $t$ period

$I P_{1}(t) \quad$ Stock level at the beginning of $t$ period, which include on-road stock quantity
$I L_{2}(t) \quad$ On-hand stock quantity at the end of $t$ period

$D(t) \quad$ Demand quantity at $t$ period

$B L(t) \quad$ Back order quantity at $t$ period quantity, which means stock out happens at the time

$D S(t) \quad$ Demand meeting quantity at $t$ period

$A_{Q} \quad$ Regular order cost per time

$A_{E} \quad$ Emergency order cost per time

$H \quad$ Holding cost per SKU in one period

$B \quad$ Backorder cost per SKU in one period

$p \quad$ Sale price per SKU

$S_{u}(t) \quad$ Target buffer stock level

$S_{d}(t) \quad$ Safety buffer stock level

$S_{T}(t) \quad S$ value while using $(S, S, T)$ control policy

$S_{T}(t) \quad s$ value while using $(S, s, T)$ control policy

We consider a discrete inventory control system in the model, and sequences of events in each period are: (a) Order receipts; (b) Inventory verification, including on-hand stock, on-road stock and stock level as well; (c) Demand forecasting; (d) Place the order; (e) Meet the demand, the shortage quantity would become backorder and needs to be delivered next time; (f) Inventory verification again and calculate the throughput (net profit). As shown in Fig1:

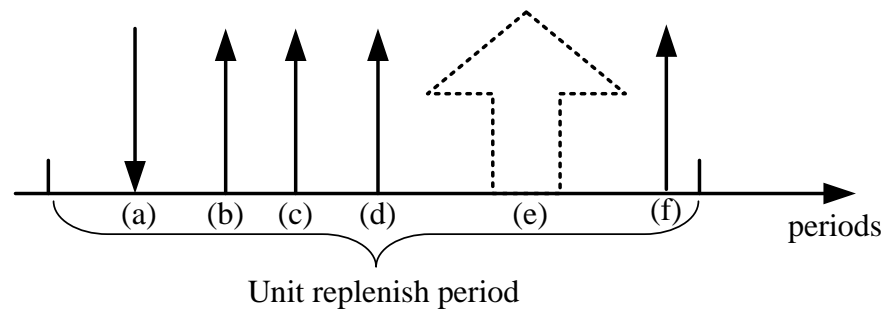

Fig.1. Event sequence of the inventory control system

Based on the Notation and assumptions above, as well as the event sequence in the inventory control system settings, after certain periods, we have:

$$
R(t)=O_{Q}\left(t-L_{Q}\right)+O_{E}\left(t-L_{E}\right)
$$

The function of on-hand stock is:

$$
I L_{1}(t)=I L_{1}(t-1)+R(t)-D(t-1)
$$

As for the back order, we have:

$$
B L(n)=\left(D(n)-I L_{1}(n)\right)^{+}
$$

In which:

$(x)^{+}=\max \{x, 0\},(x)^{-}=\max \{-x, 0\}$

Thus, $B L(t)>0$ indicates that stock out happens in the period, and the demand meeting quantity is:

$$
D S(t)=\min \left\{D(t)+B L(t-1), I L(t)^{+}\right\}
$$


On-road stock is another important factor in the model, with its function:

$$
I T(t)=\sum_{k=1}^{L_{Q}} O_{Q}(t-k)+\sum_{k=1}^{L_{E}} O_{E}(t-k)
$$

Then we have the stock level at the beginning of at $t$ period:

$$
I P_{1}(t)=I L_{1}(t)+I T(t)
$$

While we calculate the throughput of the whole system, average throughput in $\mathrm{N}$ periods will be considered:

$$
T P(N)=T R(N)-O E(N)-T C(N)
$$

In which $T R(N)$ stands for the average sales value; $O E(N)$ stands for the ordering cost, which include regular order cost and emergency order cost; $T C(N)$ stands for the total holding cost, which include both holding cost and back order cost:

$$
T C(N)=\sum_{t=1}^{N} \frac{1}{N}\left(H \times I L(t)^{+}+B \times I L(t)^{-}\right)
$$

Total cost obviously depends on the order frequency and quantity each period. Sometimes throughput $T P(N)$ could be considered as the net profit of the system, which will be calculated as:

$$
\operatorname{TR}(N)=\frac{1}{N} \sum_{t=1}^{N} p D S(t)
$$

The basic control procedure is as same as the genetic buffer management framework proposed in Ref. 6 . Monitoring window (MW) mechanism also has been set in our model, which is still a time interval that functions

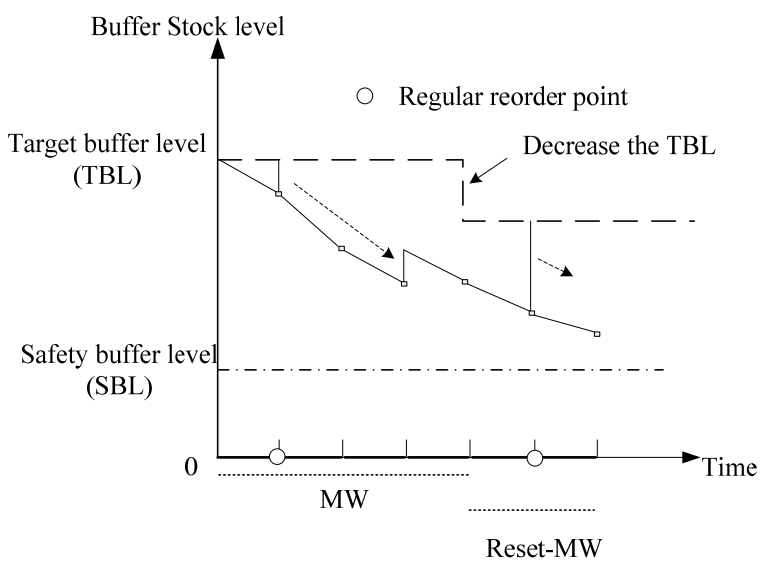

as a reference for tracking the buffer consumption status and deciding which action must be taken. The monitoring window should be higher than the average replenishment time between shipments. The monitoring window is reset under the following conditions. (1) During the monitoring window, no safety buffer penetration occurs; the green buffer is adjusted and a new monitoring window is reset at the end of the previous monitoring window. (2) Whenever either the safety buffer level or the target buffer level is adjusted during the monitoring window, the monitoring window is reset from the period which follows the one in which the buffer was adjusted. Whenever the monitoring window is reset, the buffer consumption status of the previous monitoring window is excluded from the decision regarding this new monitoring window.

The inventory control system operates with following rules:

(1) Target buffer stock level adjusts rules.

Penetration of the safety buffer is first checked for, if no penetration has occurred, then if the monitoring window has ended, then the target buffer stock level is decreased, the monitoring window is reset and the next monitor is initiated. The green buffer level is reduced because the lack of safety buffer penetration implies that the target buffer stock level is too high. Meanwhile, If stock out does occur, an emergency order is placed and the target buffer level is increased because the target buffer stock level is too small. Again, the monitoring window will be reset and the next monitor initiated since the target buffer stock level has also been adjusted, as shown in Fig2.

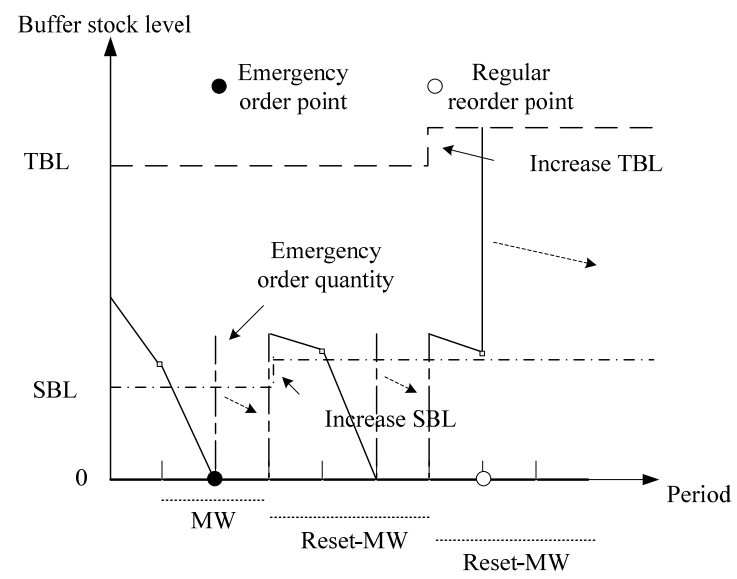

Fig.2. Target buffer stock level adjust procedure (Source Ref. 6)

(2)Safety buffer stock level adjusts rules.

If the safety buffer is penetrated, but this does not represent the second penetration of the safety buffer within the monitoring window, we check if the stock out occurs. If stock out does not occur, only the emergency replenishment order is triggered, if stock out does occur, an emergency replenishment order of the same amount is triggered but the safety buffer level is increased 
because both the green and the safety buffer levels are set too low.

Adjusting the safety buffer level allows the monitoring window to be reset and the next monitor to be initiated. As stated above, the actual size of the buffer is not critical as long as the buffer status continues to be monitored. Therefore, the extent to which the safety or green buffer levels should be increased or decreased is also subjective. If the safety buffer is penetrated but it represents the second penetration of the safety buffer within the monitoring

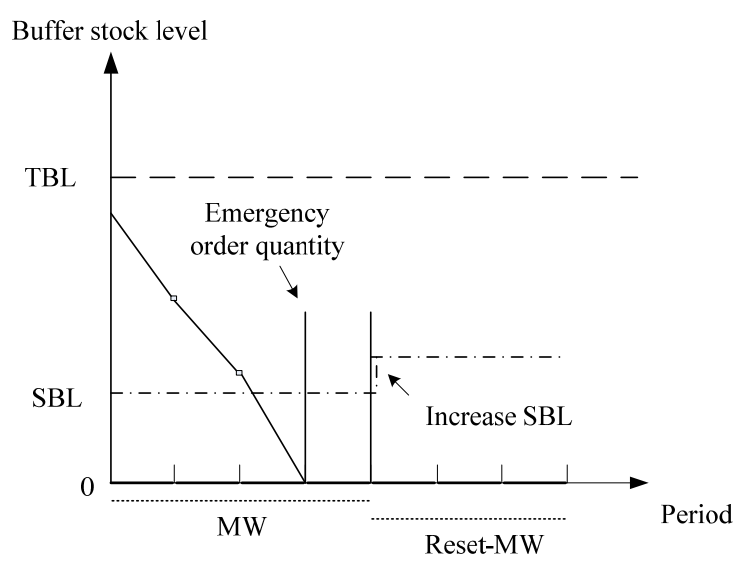

window, we check if the stock out occurs. If stock out has not occurred, then an emergency replenishment order is replaced and the safety buffer level is decreased, because in two cases of penetration, the safety buffer level may have been set too high, as shown in Fig3. Again, the monitoring window will be reset and the next monitor initiated, since the safety buffer level has been adjusted. The entire adjust procedure of the inventory control system could be simply demonstrated in Fig4.

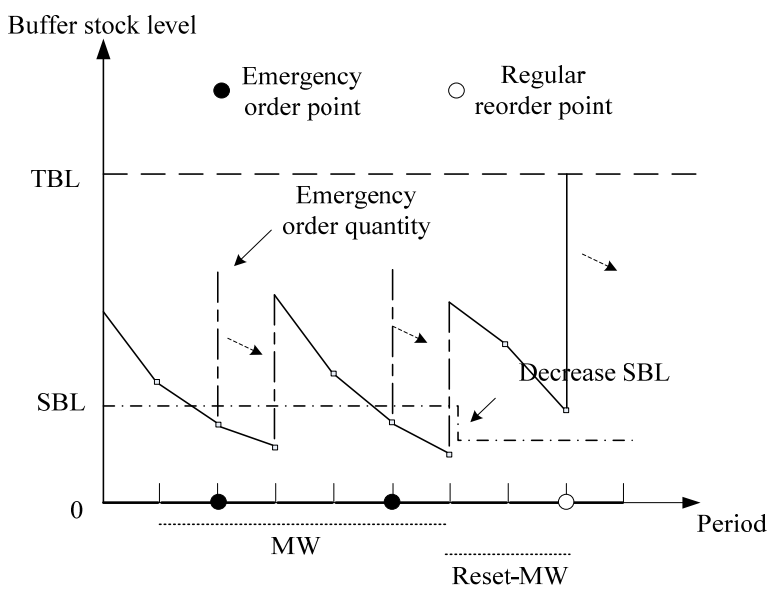

Fig. 3. Safety buffer stock level adjust procedure (Source Ref. 6)

(3)Order rules.

Every $\mathrm{T}$ periods, there would have a regular order, the order quantity set as:

$$
O_{Q}(t)=\left\{\begin{array}{l}
S_{u}(t)-I L_{1}(t), T>L \\
S_{u}(t)-I P_{1}(t), T \leq L
\end{array}\right.
$$

Meanwhile, every penetration into the safety buffer would trigger an emergency order, with its quantity set as:

$$
O_{E}(t)=\frac{S_{u}(t)+S_{d}(t)}{2}
$$

Differ from Ref. 6, we consider that the purpose of emergency order is to regain the on-hand stock level to safety stock level as soon as possible. Thus we set the quantity of each emergency order as:

$$
O_{E}(t)=S_{d}(t)-I L_{1}(t)
$$

It's obviously that the dynamic adjust procedure would great affect the performance of the system. Unfortunately, quantitive research in this area are still rare, many relative literatures are still use subjective adjust method in buffer management.

Similar to Ref. 6, first, we set the dynamic adjust procedure with randomness:

$$
\begin{aligned}
& S_{u}(t+1)=S_{u}(t) \times(1 \pm \text { rand }) \\
& S_{d}(t+1)=S_{d}(t) \times(1 \pm \text { rand })
\end{aligned}
$$

In which rand are random numbers ranges from 0-1.For simplicity, we name this inventory control policy as TOC1.

\section{Diverse Inventory Control Policies}

Based on the framework above, we proposed two novel inventory control policies.

1. With drop compensate ideology which is actually an afterwards adjust procedure, we set the adjust rules a

(1)If no penetration into safety buffer has occurred, then the monitoring window has ended, and the target buffer stock level is decreased as:

$S_{u+1}(t)=S_{u}(t)-\left[\min \{I L(t-L e n), \ldots . . I L(t)\}-S_{d}(t)\right](15)$ in which Len stands for the length of the MW.

(2)If the safety buffer is penetrated, we check if the stock out occurs, and if stock out does occur, an emergency replenishment order of the same amount is triggered but the safety buffer level is increased as: 
$S_{d}(t+1)=\min \left\{S_{d}(t)+\frac{L_{Q}}{2} \times D(t-1), k S_{u}(t)\right\}$

In which $k \hat{I}(0,1)$, and it make sure that the safety buffer level would not exceed the target buffer stock level.

(3)If penetration of the safety buffer has occurred, and stock out does occur, an emergency order is placed and the target buffer level is increased as:

$$
S_{u}(t+1)=S_{u}(t)+\frac{L_{Q}}{2} \times D(t)
$$

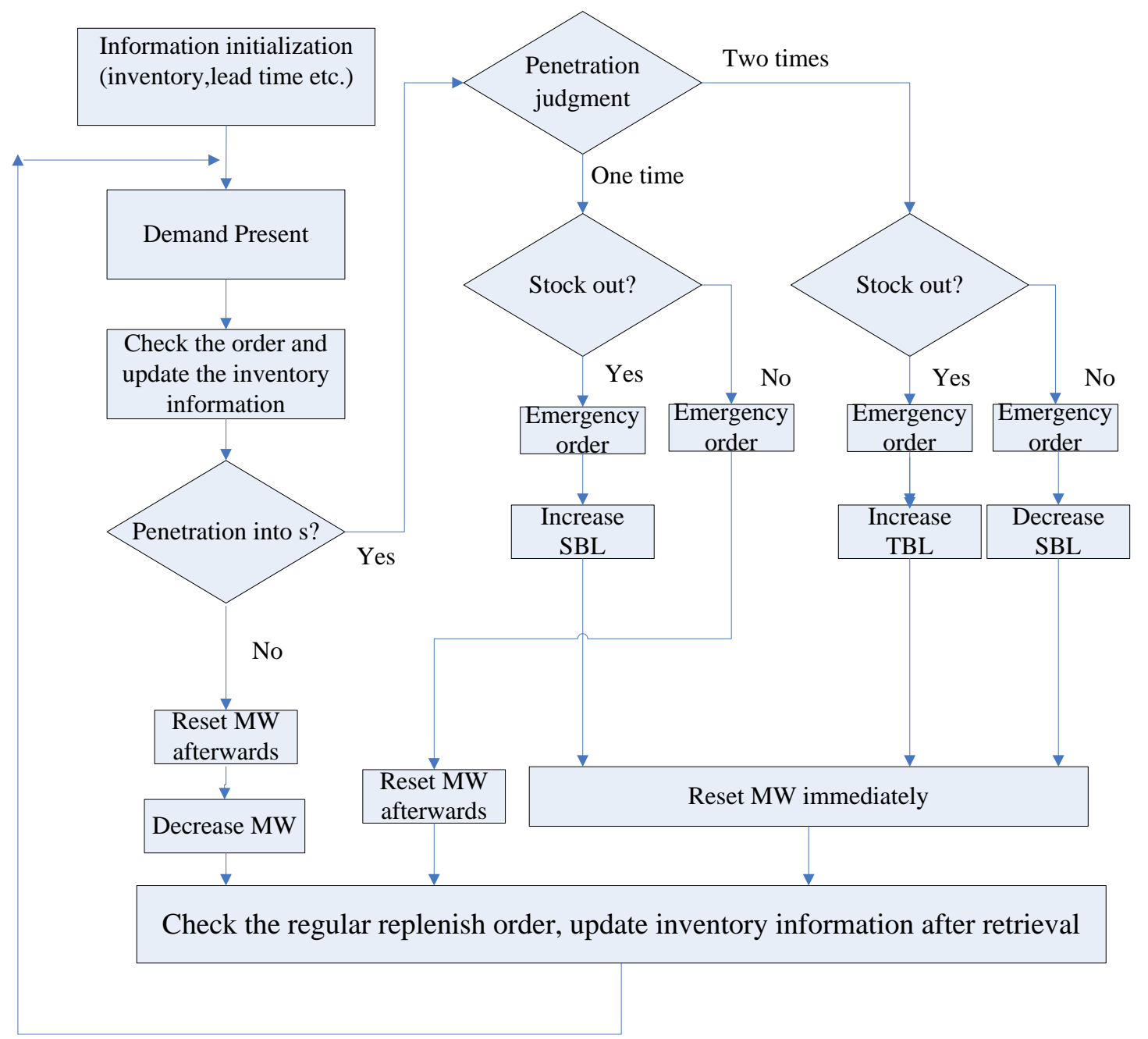

Fig.4. Adjust procedure of the inventory control system based on TOC

2. As demand forecast remains a big issue in reality, we proposed another inventory control policy based on demand forecasting. Even though there are many forecasting method being applied in reality, no matter which one has been chosen in procedure, we note
(4) If the safety buffer is penetrated and it represents the second penetration of the safety buffer within the monitoring window, and stock out has not occurred, then an emergency replenishment order is replaced and the safety buffer level is decreased as:

$S_{d}(t+1)=S_{d}(t)-\min \{I L(t-\operatorname{Len}+\operatorname{Len}(t)), \ldots I L(t)\}$

in which $\operatorname{Len}(t)$ stands for the rest time of the MW.

For simplicity, we name this inventory control policy with drop compensate ideology as TOC2. 


$$
S_{u}(t+1)=\min \left\{D_{f}(t) \times \min \left(T, L_{Q}\right)+L_{Q} D_{f}(t), S_{u}(t)\right\}(19)
$$

(2) If the safety buffer is penetrated, we check if the stock out occurs, and if stock out does occur, an emergency replenishment order of the same amount is triggered but the safety buffer level is increased as:

$$
S_{d}(t+1)=\max \left\{D_{f}(t) \times L_{Q}, S_{d}(t)\right\}
$$

(3)If penetration of the safety buffer has occurred, and stock out does occur, an emergency order is placed and the target buffer level is increased as:

$$
S_{u}(t+1)=\max \left\{D_{f}(t) \times \min \left(L_{Q}, T\right)+D_{f}(t) \times L_{Q}, S_{u}(t)\right\}
$$

(4)If the safety buffer is penetrated and it represents the second penetration of the safety buffer within the monitoring window, and stock out has not occurred, then the safety buffer level should be adjust as:

$$
S_{d}(t+1)=\min \left\{D_{f}(t) \times L_{Q}, S_{d}(t)\right\}
$$

For simplicity, we name this inventory control policy based on demand forecasting as TOC3.

Furthermore, for persuasive purpose, we introduced an adaptive $(\mathrm{s}, \mathrm{S}, \mathrm{T})$ inventory control policy in the simulation of our comparison. In which the parameters are set as:

$$
\left\{\begin{array}{l}
s_{T}(t)=L_{Q} D_{f}(t) \\
S_{T}(t)=s_{T}(t)+\min \left\{L_{Q}, T\right\} D_{f}(t)
\end{array}\right.
$$

and the order quantity $Q=S-S$ every $\mathrm{T}$ periods, other process are as same as $(\mathrm{s}, \mathrm{S})$ policy.

\section{Simulation and Analysis}

As nonstationary demand character often appear as short time demand fluctuation in reality. And ARIMA model has shown its efficiency in short period forecasting, thus, we implement ARIMA in our simulation for demand forecast module, then we have to use exponential smoothing method to eliminate the seasonal effect:

$$
D_{f}(t)=D_{f}(t-1)+(1-\theta)\left(D(t-1)-D_{f}(t-1)\right)
$$

In which $q$ stands for the prediction coefficients.

And we use following function to simulate the demand fluctuation:

$\left\{\begin{array}{c}D(1)=\mu+\varepsilon_{1} \\ D(t)=\mu+\alpha_{t} D(t-1)-(1-\gamma) \varepsilon_{t-1}+\varepsilon_{t}\end{array}, 0 \leq \alpha_{t}<1,0<\gamma<1\right.$

In which $\alpha(t)=\left|\sin \left(\frac{\pi t}{12}\right)\right|$ stand for seasonal coefficients, $m$ and $g$ are objective parameters for different market environment, $\left\{\varepsilon_{t}\right\}$ is the white noise, and $\operatorname{Mean}(\varepsilon(t))=0$

, $\operatorname{Var}(\varepsilon(t))=\sigma^{2}$ in our simulation, we set the relative parameters as: $\sigma^{2}=100, \mu=20, \quad \theta=0.6$, $\gamma=0.6$. The simulation is running with 120 sale periods, without loss of generality, demand fluctuation in the last 20 periods are steady and smooth, as shown in Fig5.

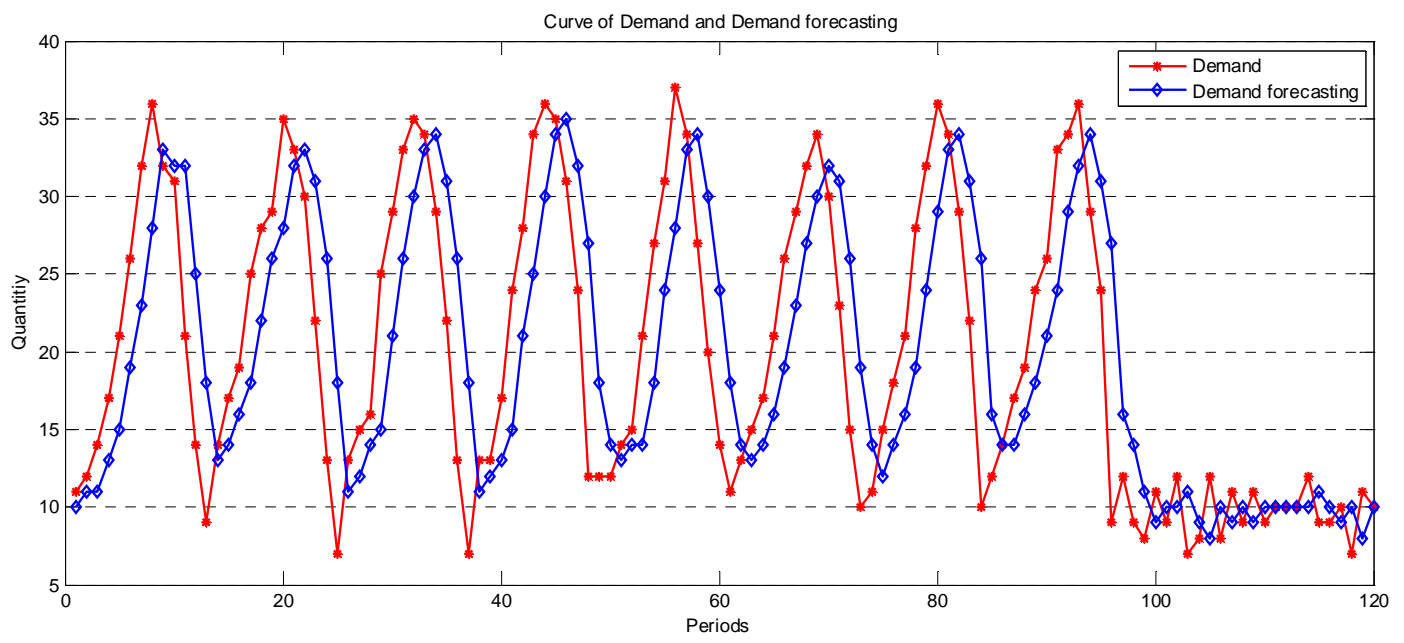

Fig.5. Curve of demand and demand forecasting

Other important parameters for TOC control policies are set as: $L_{Q}=3, L_{E}=1, T=5, T_{M W}=6$ and for adaptive(s,S,T)policy, we set the replenish reorder check period as same as the TOC policy. The comparison of on-hand stock variation for different inventory control policies are shown in Fig6-Fig9 From the Figures, we found that TOC3 and $(\mathrm{s}, \mathrm{S}, \mathrm{T})$ policy performed better than the other two 


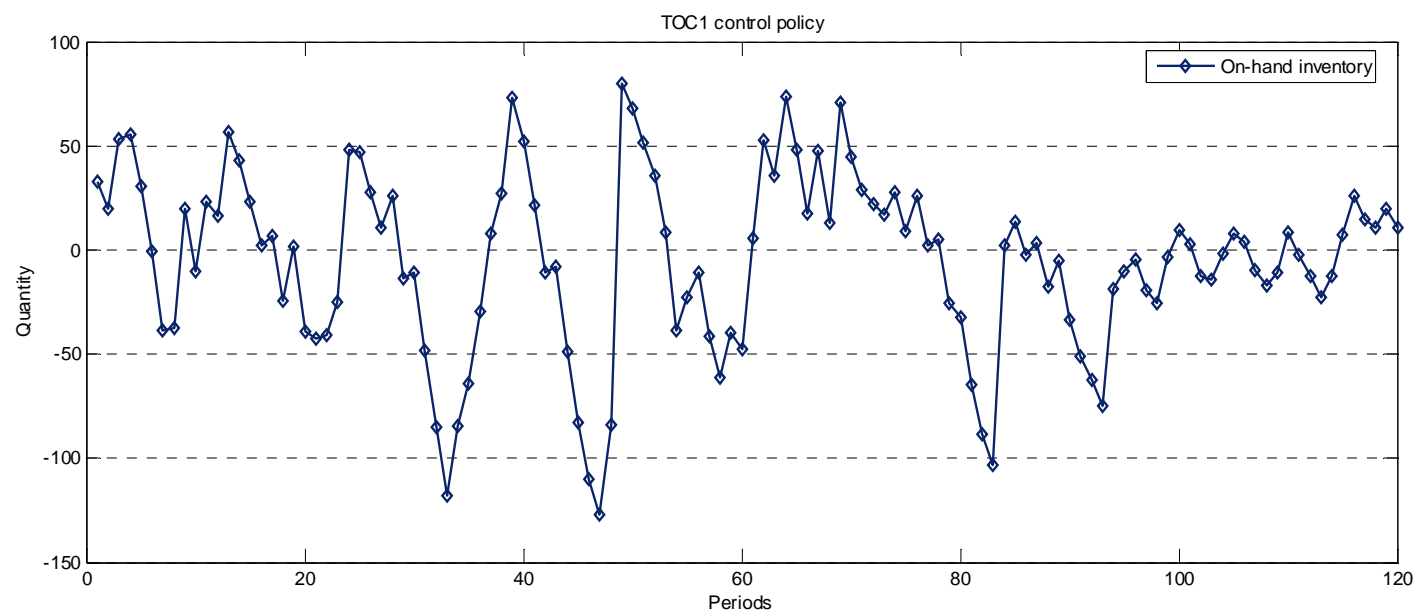

Fig.6. On-hand inventory curve of TOC1 control policy

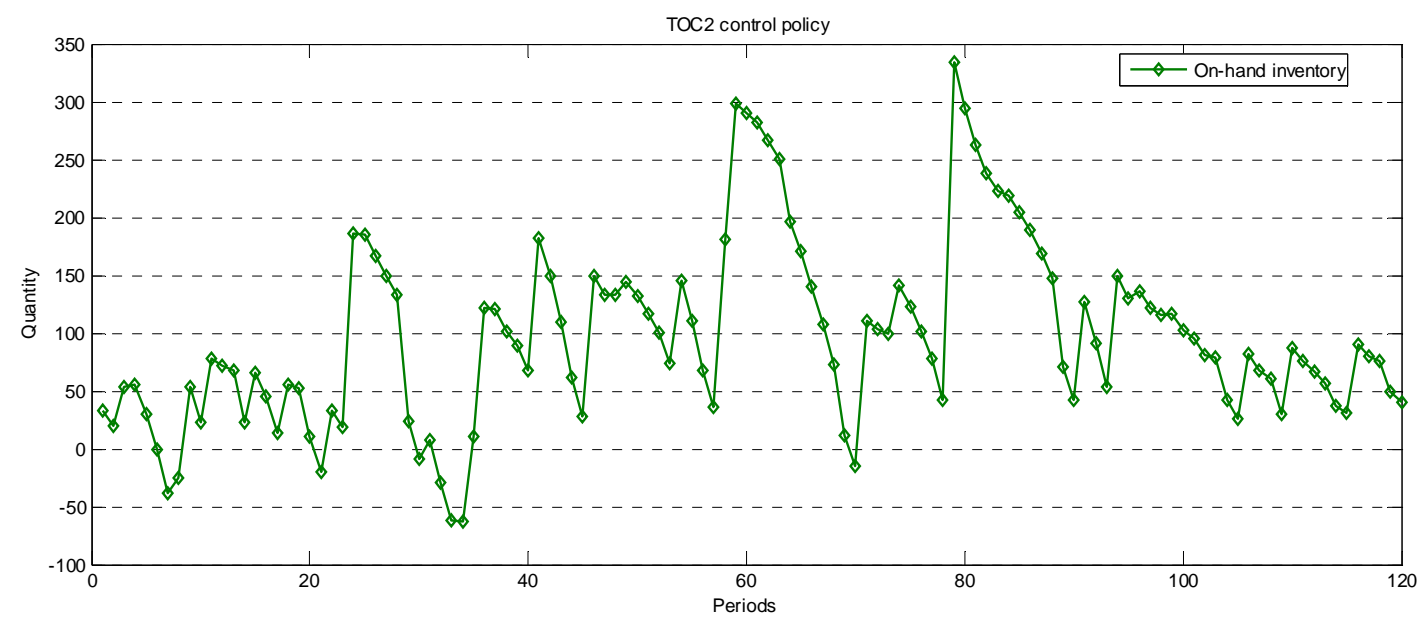

Fig.7. On-hand inventory curve of TOC2 control policy

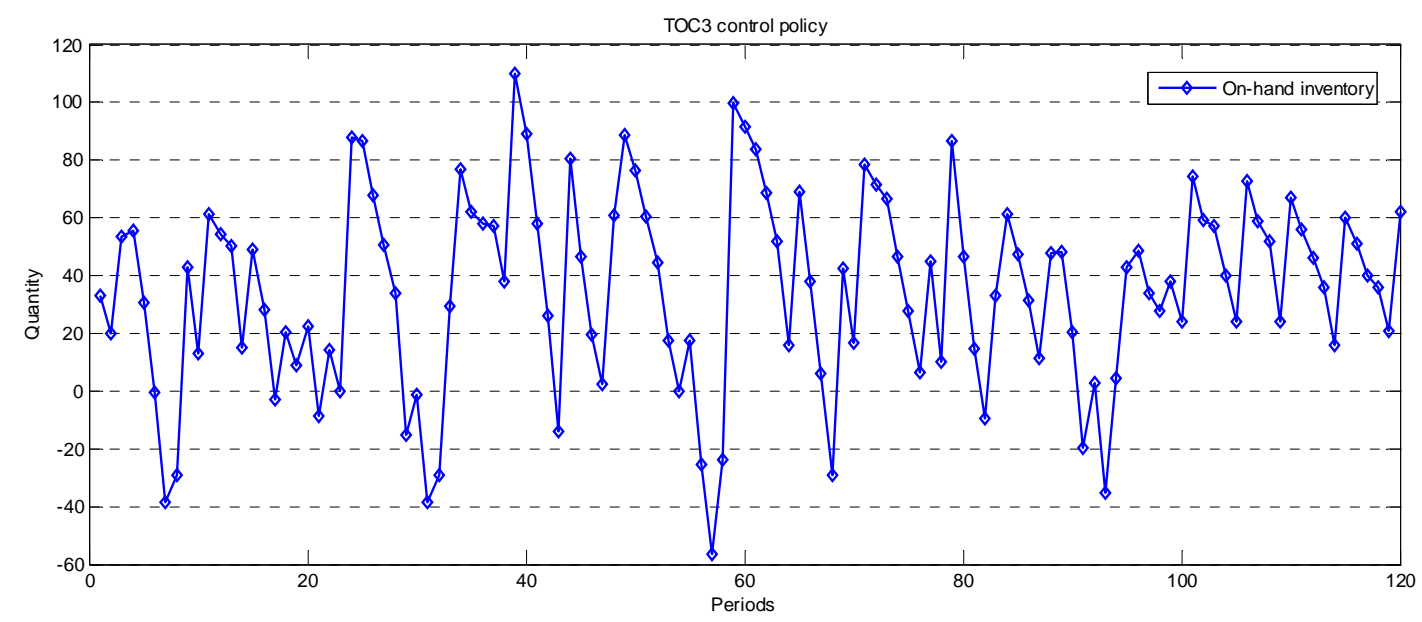

Fig.8. On-hand inventory curve of TOC3 control policy 


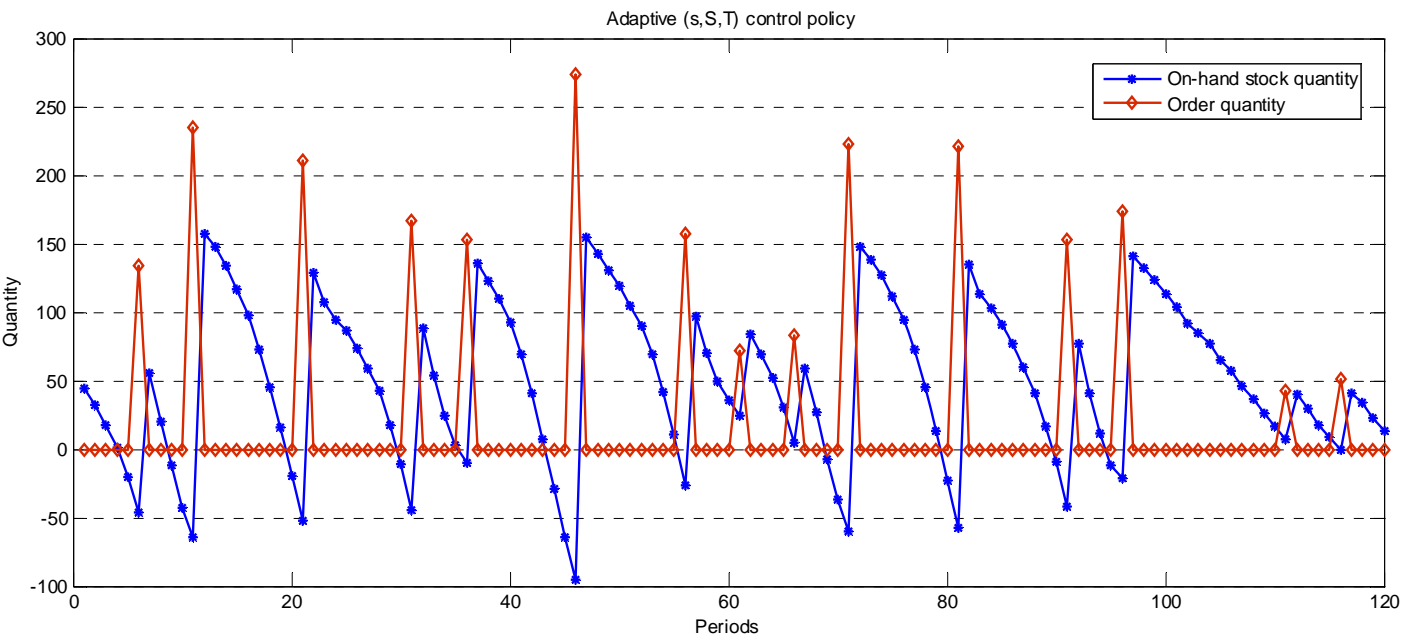

Fig.9. On-hand inventory and order curve of adaptive (s,S,T) control policy

policies, however, the on-hand stock variation has limit impact on the performance of the whole system.

The performance of backorders and demand meeting are shown in Fig10-Fig13, from which we found that TOC2 and TOC3 policy performed better than the other two, which means better service level could be provided. However, we still have to notice that the high service level provided by TOC2 policy is based on a higher inventory level. The adjust process of each policies are shown in Fig14-Fig17, and TOC3 policy has shown its stability and robust character, and adaptive (s,S,T) policy has shown its great reliability on forecasting accuracy which may not so countable in reality.

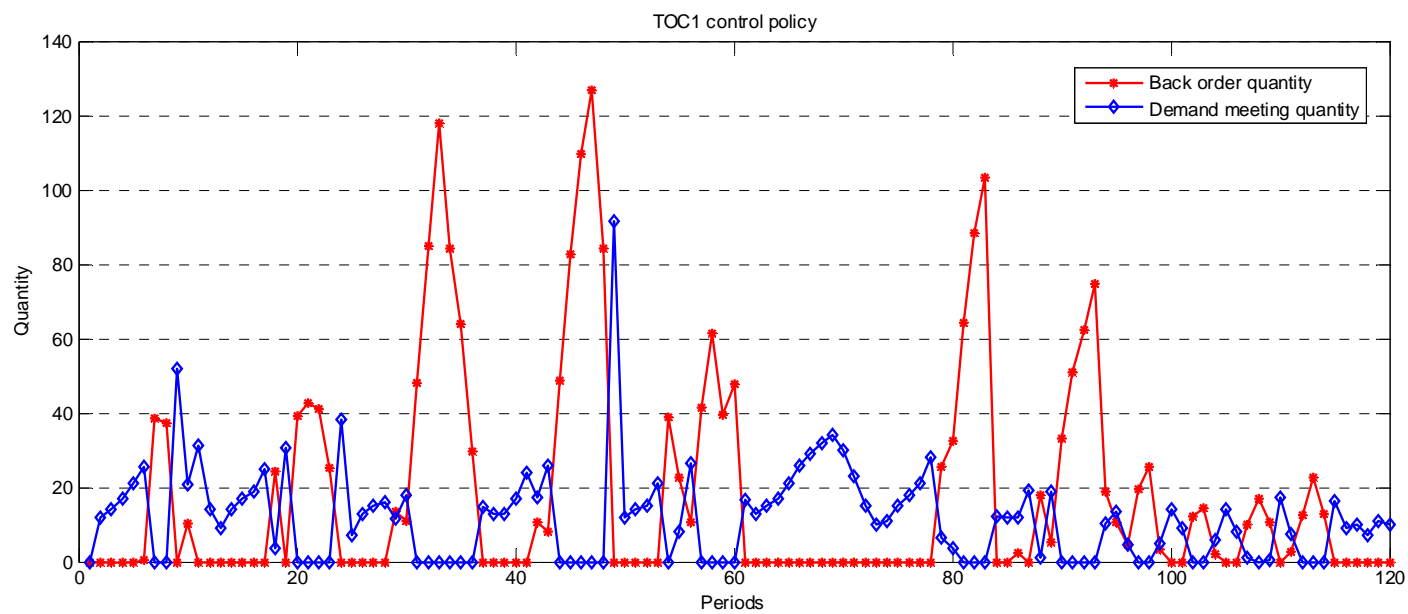

Fig.10. Back order and Demand meeting curve of TOC1 control policy 


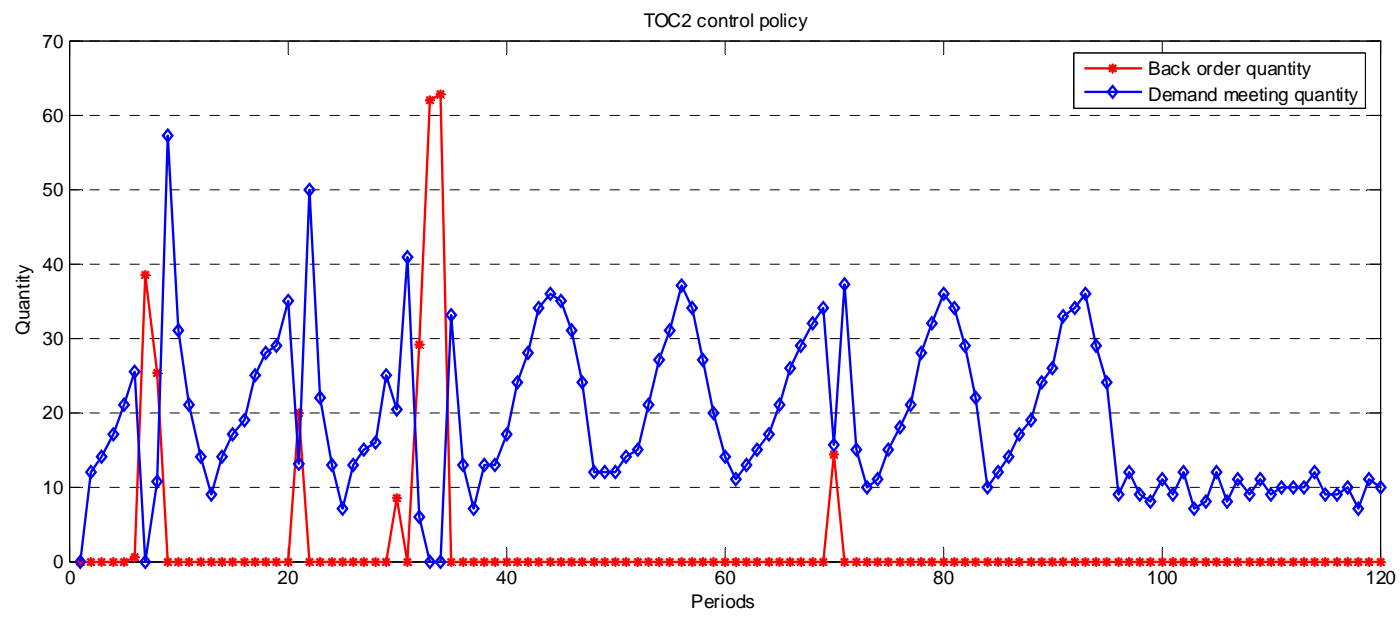

Fig.11. Back order and Demand meeting curve of TOC2 control policy

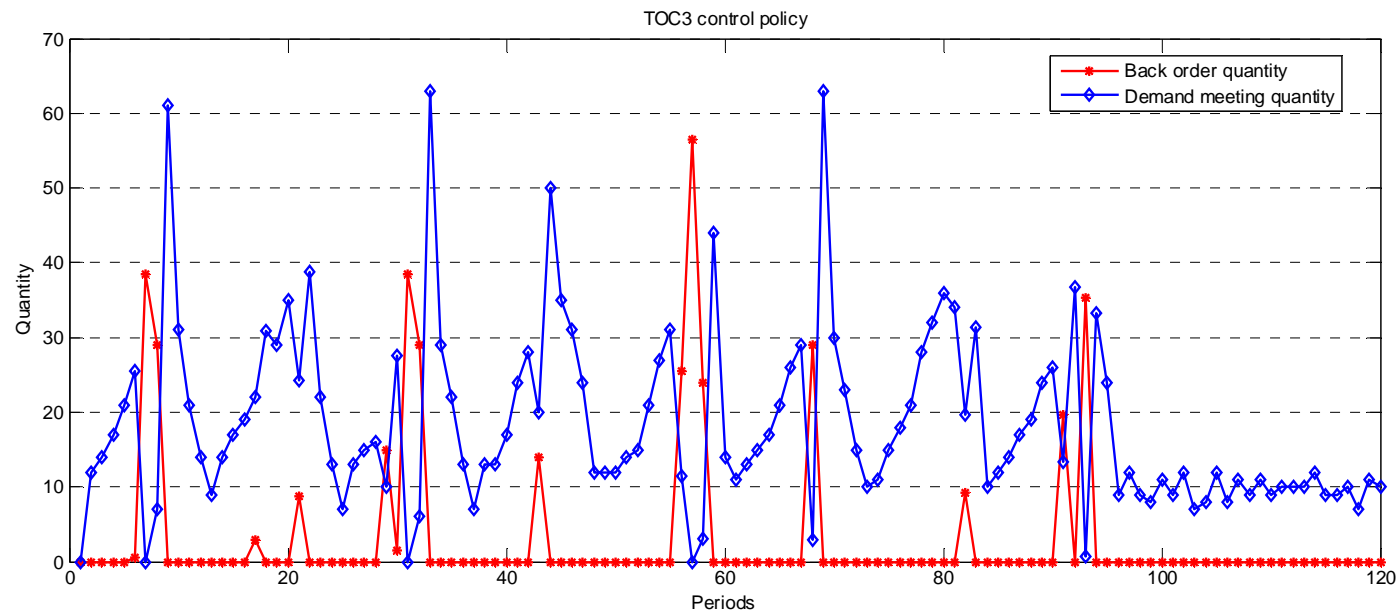

Fig.12. Back order and Demand meeting curve of TOC3 control policy

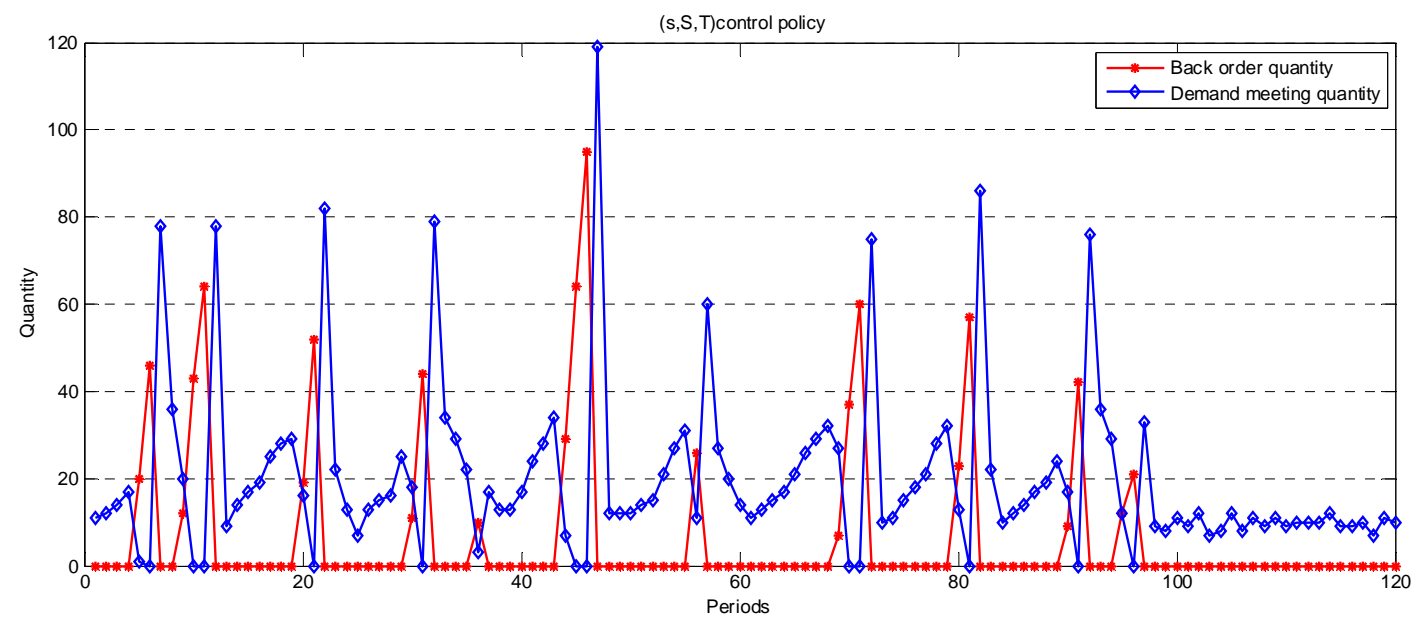

Fig.13. Back order and Demand meeting curve of adaptive (s,S,T) control policy 


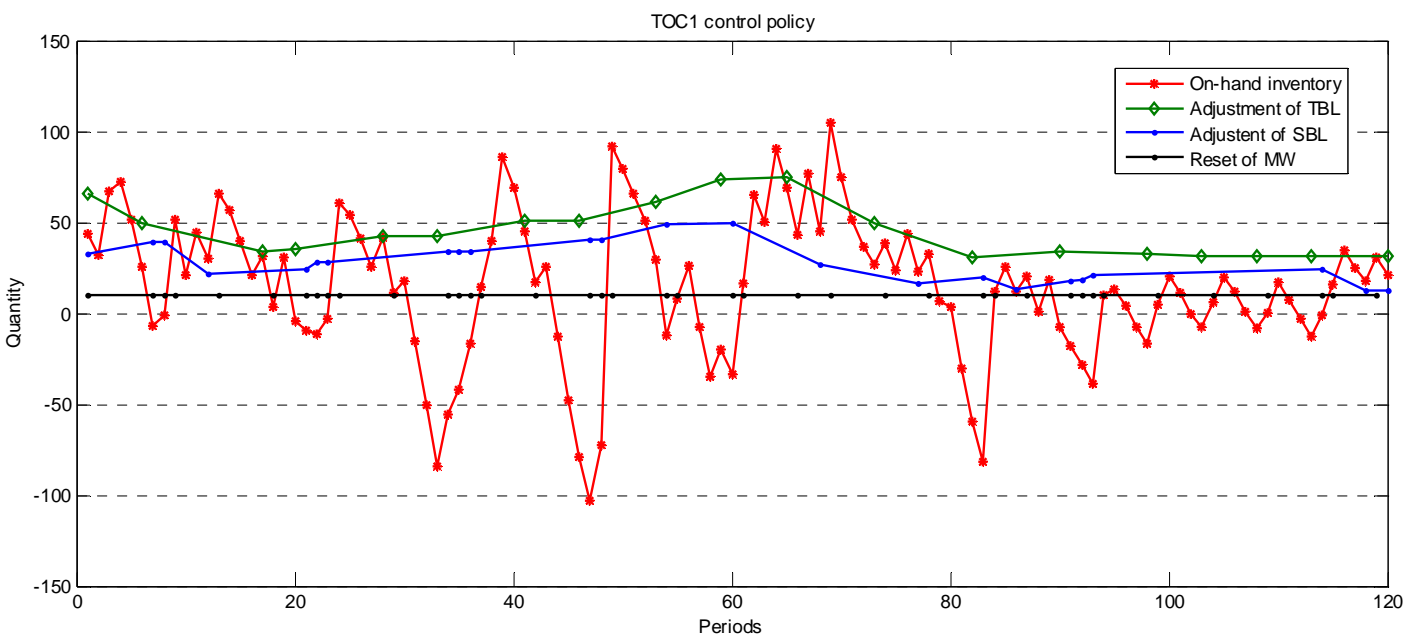

Fig.14. Inventory adjustment process under TOC1 control policy

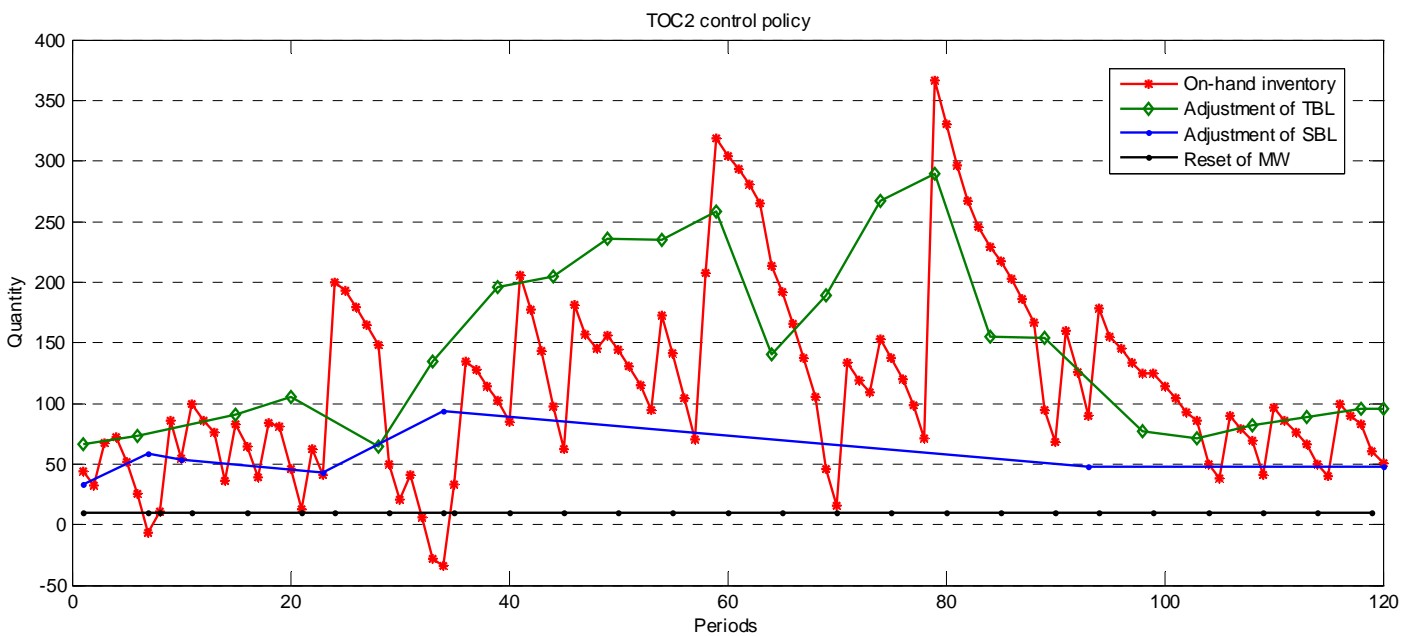

Fig.15. Inventory adjustment process under TOC2 control policy

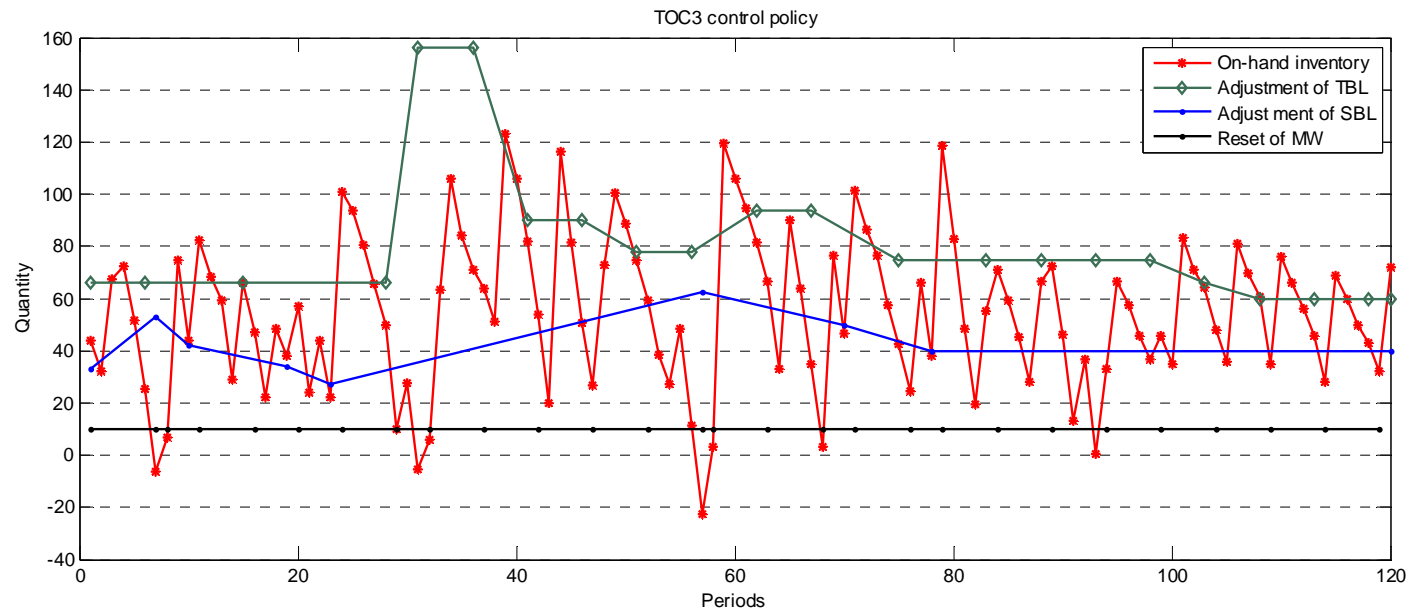

Fig.16. Inventory adjustment process under TOC3 control policy 


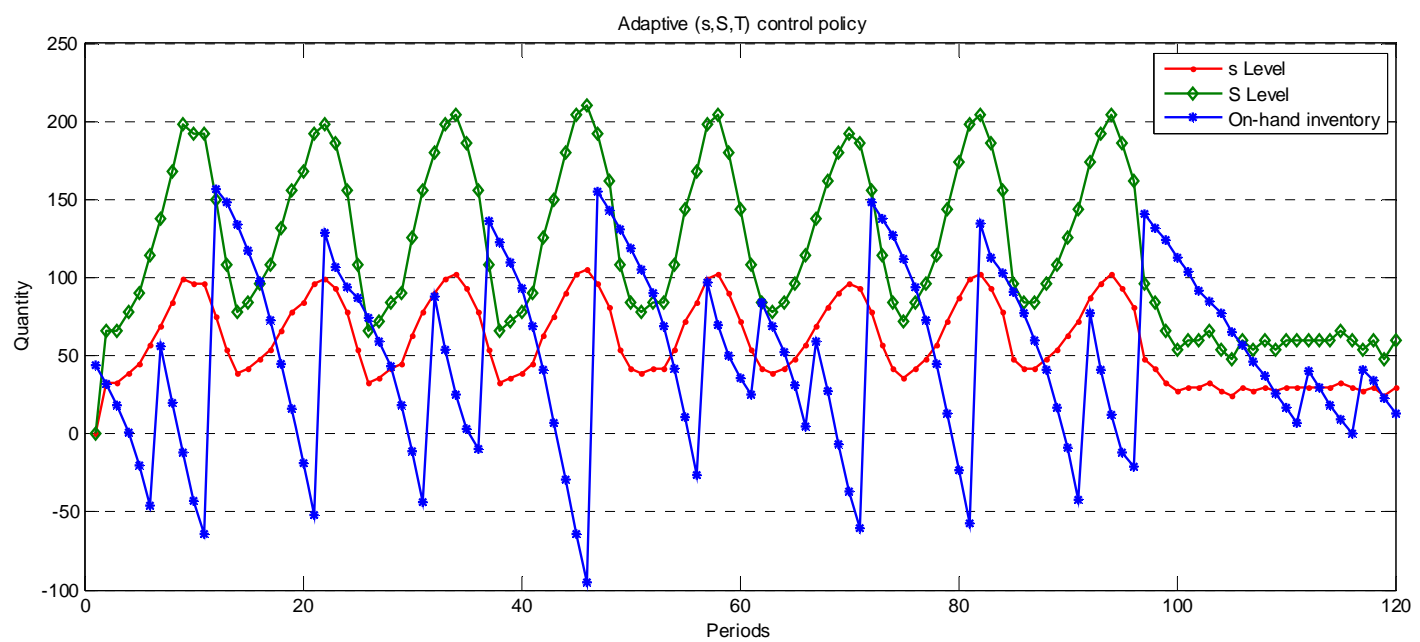

Fig.17. Inventory adjustment process under adaptive (s,S,T) control policy

As for the performance evaluation, we consider holding cost, order cost and throughput as our main index. Other important parameters are set as: $A_{Q} \in[100,160], A_{E}=200, H \in[2,5], p=40$, $B=40$. Still, for adaptive $(\mathrm{s}, \mathrm{S}, \mathrm{T})$ policy , we set $H \in[1,9]$.

Based on the parameters we input above, the simulation output based on proposed TOC control policies are shown in Table1-Talbe3,from which we can conclude that TOC1 policy keep a low inventory level, however, with backorder happens a lot, which causes too much shortage cost and leads to a low throughput value. TOC2 keeps a high service level based on a high inventory level, and we can discover that TOC2 policy are not sensitive to regular order cost $A_{Q}$ compared with the holding cost $H$, which means throughput will drop rapidly with the raise of holding cost. TOC3 policy keeps a proper service level and inventory level, and exhibit no sensitive to regular order cost $A_{Q}$ and the holding cost $H$, thus it guarantees both service level and the throughput of the system. Meanwhile, from Table4 we can conclude that adaptive $(\mathrm{s}, \mathrm{S}, \mathrm{T})$ policy are much more sensitive to holding cost $H$ compared with the regular order cost $A_{Q}$, in conclusion, TOC3 performed best among all the policies we proposed in this paper.

Table 1 Performance of TOC1 control policy

\begin{tabular}{|c|c|c|c|c|c|c|c|c|c|c|}
\hline$A_{Q}$ & $A_{E}$ & $H$ & $B$ & $p$ & $\begin{array}{l}\text { Total holding } \\
\text { cost }\end{array}$ & $\begin{array}{l}\text { Total backorder } \\
\text { cost }\end{array}$ & $\begin{array}{l}\text { Regular order } \\
\text { cost }\end{array}$ & $\begin{array}{l}\text { Emergency order } \\
\text { cost }\end{array}$ & Sales & Throughput \\
\hline 100 & 200 & 2 & 40 & 40 & 68.2109 & 452.4417 & 12.5000 & 68.3333 & 541.5764 & -59.9096 \\
\hline 120 & 200 & 2 & 40 & 40 & 68.2109 & 452.4417 & 15.0000 & 68.3333 & 541.5764 & -62.4096 \\
\hline 140 & 200 & 2 & 40 & 40 & 68.2109 & 452.4417 & 17.5000 & 68.3333 & 541.5764 & -64.9096 \\
\hline 160 & 200 & 2 & 40 & 40 & 68.2109 & 452.4417 & 20.0000 & 68.3333 & 541.5764 & -67.4096 \\
\hline 100 & 200 & 3 & 40 & 40 & 102.3164 & 452.4417 & 20.0000 & 68.3333 & 541.5764 & -101.5150 \\
\hline 100 & 200 & 4 & 40 & 40 & 136.4218 & 452.4417 & 20.0000 & 68.3333 & 541.5764 & -135.6205 \\
\hline 100 & 200 & 5 & 40 & 40 & 170.5273 & 452.4417 & 20.0000 & 68.3333 & 541.5764 & -169.7259 \\
\hline
\end{tabular}

Table 2 Performance of TOC2 control policy

\begin{tabular}{|c|c|c|c|c|c|c|c|c|c|c|}
\hline$A_{Q}$ & $A_{E}$ & $H$ & $B$ & $p$ & $\begin{array}{l}\text { Total holding } \\
\text { cost }\end{array}$ & $\begin{array}{l}\text { Total backorder } \\
\text { cost }\end{array}$ & $\begin{array}{l}\text { Regular order } \\
\text { cost }\end{array}$ & $\begin{array}{l}\text { Emergency order } \\
\text { cost }\end{array}$ & Sales & Throughput \\
\hline 100 & 200 & 2 & 40 & 40 & 198.5150 & 86.9667 & 10.0000 & 35.0000 & 755.9917 & 425.5100 \\
\hline 120 & 200 & 2 & 40 & 40 & 198.5150 & 86.9667 & 12.0000 & 35.0000 & 755.9917 & 423.5100 \\
\hline 140 & 200 & 2 & 40 & 40 & 198.5150 & 86.9667 & 14.0000 & 35.0000 & 755.9917 & 421.5100 \\
\hline 160 & 200 & 2 & 40 & 40 & 198.5150 & 86.9667 & 16.0000 & 35.0000 & 755.9917 & 419.5100 \\
\hline 100 & 200 & 3 & 40 & 40 & 297.7725 & 86.9667 & 20.0000 & 35.0000 & 755.9917 & 316.2525 \\
\hline 100 & 200 & 4 & 40 & 40 & 397.0300 & 86.9667 & 20.0000 & 35.0000 & 755.9917 & 216.9950 \\
\hline 100 & 200 & 5 & 40 & 40 & 496.2875 & 86.9667 & 20.0000 & 35.0000 & 755.9917 & 117.7375 \\
\hline
\end{tabular}


Table 3 Performance of TOC3 control policy

\begin{tabular}{|c|c|c|c|c|c|c|c|c|c|c|}
\hline$A_{Q}$ & $A_{E}$ & $H$ & $B$ & $p$ & $\begin{array}{l}\text { Total holding } \\
\text { cost }\end{array}$ & $\begin{array}{l}\text { Total backorder } \\
\text { cost }\end{array}$ & $\begin{array}{l}\text { Regular order } \\
\text { cost }\end{array}$ & $\begin{array}{l}\text { Emergency order } \\
\text { cost }\end{array}$ & Sales & Throughput \\
\hline 100 & 200 & 2 & 40 & 40 & 76.3089 & 125.6139 & 14.1667 & 51.6667 & 730.8339 & 463.0777 \\
\hline 120 & 200 & 2 & 40 & 40 & 76.3089 & 125.6139 & 17.0000 & 51.6667 & 730.8339 & 460.2444 \\
\hline 140 & 200 & 2 & 40 & 40 & 76.3089 & 125.6139 & 19.8333 & 51.6667 & 730.8339 & 457.4111 \\
\hline 160 & 200 & 2 & 40 & 40 & 76.3089 & 125.6139 & 22.6667 & 51.6667 & 730.8339 & 454.5777 \\
\hline 100 & 200 & 3 & 40 & 40 & 114.4634 & 125.6139 & 20.0000 & 51.6667 & 730.8339 & 419.0899 \\
\hline 100 & 200 & 4 & 40 & 40 & 152.6179 & 125.6139 & 20.0000 & 51.6667 & 730.8339 & 380.9355 \\
\hline 100 & 200 & 5 & 40 & 40 & 190.7723 & 125.6139 & 20.0000 & 51.6667 & 730.8339 & 342.7810 \\
\hline
\end{tabular}

Table 4 Performance of adaptive (s,S,T) control policy

\begin{tabular}{lllllllll}
\hline$A_{Q}$ & $H$ & $B$ & $p$ & Total holding cost & Total backorder cost & Regular order cost & Sales & Throughput \\
\hline 100 & 1 & 40 & 40 & 55.3083 & 267.6667 & 12.5000 & 797.6667 & 462.1917 \\
120 & 1 & 40 & 40 & 55.3083 & 267.6667 & 15.0000 & 797.6667 & 459.6917 \\
140 & 1 & 40 & 40 & 55.3083 & 267.6667 & 17.5000 & 797.6667 & 457.1917 \\
160 & 1 & 40 & 40 & 55.3083 & 267.6667 & 20.0000 & 797.6667 & 454.6917 \\
100 & 5 & 40 & 40 & 276.5417 & 267.6667 & 12.5000 & 797.6667 & 240.9583 \\
100 & 7 & 40 & 40 & 387.1583 & 267.6667 & 12.5000 & 797.6667 & 130.3417 \\
100 & 9 & 40 & 40 & 497.7750 & 267.6667 & 12.5000 & 797.6667 & 19.7250 \\
\hline
\end{tabular}

\section{Conclusion}

We proposed three different inventory control policies for nonstationary demand environment in this paper which based on TOC generic replenishment method. With the monitoring window review of the inventory target level and red buffer level, the procedure rigorously defined a means of sizing the buffer, monitoring it and correcting it when necessary adaptively. And we use simulation method to reveal the feasibility of the procedure. Adopting the proposed procedure in a specific application environment is not difficult if the variables are defined according to the application environment. Furthermore, through the comparison, we've showed that one of the proposed buffer management procedures outperforms on prior generic buffer control management in Ref.6 and adaptive (s,S,T) policy.

Though the forecasting accuracy is not a big issue in the discussion of our paper, it's an important aspect which may greatly impact the performance of the inventory system, and then the further research could be focused in this area.

\section{References}

1. Beamon, B. M. . Supply chain design and analysis: Models and methods. International Journal of Production Economics, 55(3) (1998)pp 281 - 294.

2. Silver, E. A., Pyke, D. F., \& Peterson, R. . Inventory management and production planning and scheduling (3rd ed.).,John Wiley \& Sons Inc. 1998

3. O' Donnell, T., Maguire, L., Mcivor, R., \& Humphreys, P.. Minimizing the bullwhip effect in a supply chain using genetic algorithms. International Journal of Production Research, 44(8) (2006)pp 1523 - 1543.

4. Simatupang, T. M., Wright, A. C., \& Sridharan, N. . Applying the theory of constraints to supply chain collaboration. Supply Chain Management: An International Journal, 9(1)(2004)pp 57 - 70.

5. Horng-Huei Wu, Ching-Piao Chen, Chih-Hung Tsai, TaiPing Tsai . A study of an enhanced simulation model for TOC supply chain replenishment system under capacity constraint. Expert Systems with Applications ,37(2010) pp $6435-6440$

6. Kuo-Jung YLJan. Sheng-Hung Chang and Rong-Kwei Li. Enhancement of Theory of Constraints replenishment using a novel generic buffer management procedure. International Journal of Production Research,41(4)(2003) pp 725-740

7. Lee, H. L., S. Nahmias.. Single product, single-location models. S. C. Graves, Rinnooy A. H. G. Kan, P. H. Zipkin, eds. Handbooks in Operations Research and Management Science: Logistics of Production and Inventory, Volume 4. Elsevier Science, Amsterdam,The Netherlands, (1993)pp 1 - 55.

8. Hadley, G., T. M. Whitin.. An optimal final inventory model. Management Science. 7(1961)pp 179 - 183.

9. Karlin, Samuel. Dynamic inventory policy with varying stochastic demands. Management Science.,6(3) (1960)pp231 - 258

10. Song, J., Paul Zipkin,. Managing inventory with the prospect of obsolescence.Operation. Research. 44(1) (1996)pp 215 - 222.

11. Gallego, Guillermo, Jennifer K. Ryan, David SimchiLevi. Minimax analysis for the discrete finite horizon inventory model. Working paper ,(Columbia University, NewYork, NY,1996.)

12. James T. Treharne, Charles R. Sox. Adaptive Inventory Control for Nonstationary Demand and Partial 
Information. Management Science,48(5)(2002)pp 607 624

13. Goldratt, E.M., Cox, J.. The Goal. North River Press, Croton-on-Hudson, NY,1984.

14. Spencer, M.S., Cox III, J.F.. Optimum production technology (OPT) and the theory of constraints (TOC): analysis and genealogy.International Journal of Production Research, ,33(1995) pp1495-1504.

15. Koziol, D.S.. How the constraint theory improved a jobshop operation. Management Accounting ,69 (11) (1988) pp44-49.

16. Mabin, V.J., Balderstone, S.J.. The World of the Theory of Constraints: A Review of the International Literature. (St. Lucie Press, Boca Raton, 2000.)

17. Silver, E. A. Inventory control utider a probabilistic timevarying, demand pattern,A IIE Transactions.10(1978)pp 371-379,

18. Platt D. E.etc. Tractable (Q,R) heuristic models for conslrainted service levels. Management Science. 43(1997)pp 951-965.

19. Banerjle, A.etc., Heuristic production triggering mechanisms under discrete unequal inventory withdrawals. International Journal Production Economics. 45(1996) pp83-90.

20. Ernst, R. and Powell, S. G . Optimal inventory policies under service-sensitive demand, European Journal of Operational Rcseunh.S7(1995) pp316-27

21. Morton, T. E,etc. The finite horizon nonstationary stochastic inventory problem: near-myopic heuristics, computational testing. Management Science,41(3) (1995) pp34-43.

22. Anupindi, R., etc. The non-slationary stochastic leadtime inventory problem: near-myopic bounds, heuristics, and testing. Management Science, 42(1)(1996)pp24-29. 